\title{
Evaluation of the Modified Bessel Function of the Third Kind of Imaginary Orders
}

\author{
Amparo Gil,* Javier Segura, $\nmid$ and Nico M. Temme $\ddagger$ \\ * De'partame'nto de Matemáticas. Unirersidad Autónoma de Madrid, 28()+9-Madrid, Spain; \\ $\lceil$ De'pto. de Matemáticas. Universidad Carlos III de' Madrid, 28911-Le'ganés, Madrid, Spain: \\ and \$CWI, Posthus 94079, l(190 GB Amsterdam, The Netherlands \\ E-mail: jsegura@math.uc3m.es \\ Received February 2, 2001; revised July 23, 2001
}

\begin{abstract}
The evaluation of the modified Bessel function of the third kind of purely imaginary order $K_{i a}(x)$ is discussed; we also present analogous results for the derivative. The methods are based on the use of Maclaurin series, nonoscillatory integral representations, asymptotic expansions, and a continued fraction method, depending on the ranges of $x$ and $a$. We discuss the range of applicability of the different approaches considered and conclude that power series, the continued fraction method. and the nonoscillatory integral representation can be used to accurately compute the function $K_{i a}(x)$ in the range $0 \leq a \leq 200,0 \leq x \leq 100$; using a similar scheme the derivative $K_{i u}^{\prime}(x)$ can also be computed within these ranges. $20(x)$ Elseviur Sciencerusa

Ke' Words: Bessel functions; series expansions; continued fraction; nonoscillatory integral representations; asymptotic expansions.
\end{abstract}

\section{INTRODUCTION}

The modified Bessel functions of the third kind of purely imaginary order $K_{i u}(x)$ appear in the radial solution of the Schroedinger equation for exponential potentials $V(r)=V_{x}-$ $A e^{-r / a}[2,11]$, with $r$ the distance from the center of force, and in the calculation of quantum mechanical cross sections and reaction rates for electron impact excitation of ions and neutrals [2]. Also, the Dirichlet problem with boundary conditions on a wedge is solved in terms of integrals which require the evaluation of $K_{i u}(x)$ for a wide range of positive $x$ and $a$ [12]. Besides, $K_{i a}(x)$ is the kernel of the Kantorovich-Lebedev transform [12] and plays an important role as approximant in uniform asymptotic expansions of solutions of certain second-order linear differential equations $[5,6]$.

In the literature several approaches to the evaluation of $K_{i a}(x)$ can be found $([2,3$, 7, 9-11]), which are mainly based on integral representations. In [16] the $\tau$-method is used for computing the related function $K_{1 / 2+i \beta}(x)$, with $x, \beta \in(0,10]$. A major problem

398

$0(021-9991 /(12 \$ 3.5 .(0)$

(c) 2002 Elsevier Science (USA)

All rights reserved. 
when using integral representations of $K_{i a}(x)$ is the control of the relative accuracy. The function becomes exponentially small when $x$ and/or $a$ become large, and the standard integrals oscillate strongly. In [11] an approach is discussed based on shifting the contour of integration into the complex plane, which gives some control on the cancellations. We base our quadrature method on integrals obtained by the saddle point method (see [18]), which do not oscillate. Moreover, the dominant exponential terms that describe the asymptotic behavior of $K_{i u}(x)$ are explicitly present in the representations we are using.

In this paper, we discuss methods based on the use of series expansions, continued fractions, and nonoscillatory integral representations, depending on the ranges of $x$ and $a$ and the numerical efficiency of the different approaches. We develop algorithms for computing the function $K_{i u}(x)$ and $K_{i u}^{\prime}(x)$, aiming at a relative precision of nine significant digits, in the range $0 \leq a \leq 200,0 \leq x \leq 100$.

\section{COMPUTATIONAL ASPECTS}

Our method of evaluation of $K_{i a}(x)$ and $K_{i a}^{\prime}(x)$ is based on:

1. Series expansions for moderate values of $x / a$,

2. Asymptotic expansions for large values of $x$,

3. A continued fraction method for moderate values of $a / x$, and

4. Nonoscillatory integral representations where all the three previous methods fail.

Let us now describe in detail each of these approaches. Later, we will describe the regions of numerical validity and the performance of the combined algorithm.

\section{Series Expansion}

Series expansions for $K_{i u}(x)$ and $K_{i u}^{\prime}(x)$ can be built which properly handle the singularity at $x=0$. The idea, as in [19], is to relate $K_{v}(x)$ with $I_{v \prime}(x)$ by

$$
K_{1,}(x)=\frac{\pi}{2 \sin (\pi v)}\left[I_{-v}(x)-I_{1}(x)\right]
$$

and use series expansions for the $I$ functions to obtain the expression

$$
K_{k}(x)=\sum_{k=0}^{\infty} c_{k} f_{k},
$$

which together with

$$
K_{v}^{\prime}(x)=\frac{v}{x} K_{v}(x)-K_{v+1}(x)
$$

gives

$$
K_{v}^{\prime}(x)=\frac{2}{x} \sum_{k=0}^{\infty} c_{k}\left[k f_{k}-\frac{r_{k}}{2}\right]
$$

where

$$
\begin{aligned}
& f_{k}=\frac{\pi}{2 \sin \nu \pi}\left[\frac{(x / 2)^{-\prime}}{\Gamma(k+1-\nu)}-\frac{(x / 2)^{\prime \prime}}{\Gamma(k+1+\nu)}\right], \\
& r_{k}=\frac{\pi v}{2 \sin \nu \pi}\left[\frac{(x / 2)^{-\prime}}{\Gamma(k+1-\nu)}+\frac{(x / 2)^{\prime \prime}}{\Gamma(k+1+\nu)}\right],
\end{aligned}
$$


and

$$
c_{k}=\left(\frac{x^{2}}{4}\right)^{k} \frac{1}{k !}=\frac{x^{2}}{4} \frac{c_{k-1}}{k} .
$$

The following three-term recurrence relation for $r_{k}$ can be established:

$$
\left(k^{2}-v^{2}\right) r_{k}-(2 k-1) r_{k-1}+r_{k-2}=0 .
$$

The same recurrence relation applies for $f_{k}$. Perron's theorem $[8,17]$ is inconclusive with respect to the existence of minimal solutions for this recurrence. We are using such a recurrence to evaluate the coefficients $r_{k}$ and then we use

$$
f_{k}=\frac{k f_{k-1}+r_{k-1}}{k^{2}-v^{2}}=\frac{k f_{k-1}+r_{k-1}}{k^{2}+a^{2}},
$$

where we have set $v=i a$. Notice that the evaluation of the coefficients for imaginary $v$ is not so restricted as in the case of real $v$. For real $v$, singularities in Eqs. (5), (6), and (9) are present which disappear for imaginary $\nu$. For real orders, these series expansions converge for $|v|<1$ [19]; there is no such restriction for imaginary $v$.

In order to evaluate $r_{k}$ and $f_{k}$, one needs the starting values: $f_{0}, r_{0}$, and $r_{1}$. Setting $v=i a$ we have

$$
f_{0}=-\frac{\pi}{2 i \sinh \pi a}\left[\frac{(x / 2)^{i a}}{\Gamma(1+i a)}-\frac{(x / 2)^{-i a}}{\Gamma(1-i a)}\right] .
$$

Writing

$$
\Gamma(1+i a)=|\Gamma(1+i a)| e^{i \sigma_{0}(a)}, \quad \Gamma(1-i a)=|\Gamma(1-i a)| e^{-i \sigma_{0}(a)},
$$

where $\sigma_{0}(a)$ is the Coulomb phase shift [4], we get

$$
f_{0}=\sqrt{\frac{\pi}{a \sinh \pi a}} \sin \left[a \ln (x / 2)-\sigma_{0}(a)\right],
$$

and similarly

$$
\begin{gathered}
r_{0}=\sqrt{\frac{\pi a}{\sinh \pi a}} \cos \left[a \ln (x / 2)-\sigma_{0}(a)\right], \\
r_{1}=\frac{1}{1+a^{2}} \sqrt{\frac{\pi a}{\sinh \pi a}}\left\{\cos \left[\ln (x / 2)-\sigma_{0}(a)\right]+a \sin \left[a \ln (x / 2)-\sigma_{0}(a)\right]\right\} .
\end{gathered}
$$

For the computation of $\sigma_{0}$, one can use the parameterization given in [4].

\section{Asymptotic Expansion}

For large values of $x$, one has the asymptotic expansion

$$
K_{v}(x) \sim\left(\frac{\pi}{2 x}\right)^{1 / 2} e^{-x} \sum_{k=0}^{\infty} \frac{(\nu, m)}{(2 x)^{m}},
$$


where $(\nu, m)$ is the Hankel symbol given by

$$
(\nu, m)=\frac{1}{\pi m !}(-1)^{m} \cos \nu \pi \Gamma\left(\frac{1}{2}+v+m\right) \Gamma\left(\frac{1}{2}-v+m\right) .
$$

If $\nu=i a$, one has the following recursive relation for $(i a, m)$ :

$$
(i a, m+1)=-\frac{\left(m+\frac{1}{2}\right)^{2}+a^{2}}{m+1}(i a, m)
$$

This asymptotic expansion is of a more restricted applicability than in the case of real $\nu$, the reason being that the values of $(\mathrm{i} a, \mathrm{~m})$ increase more rapidly than in the case of real orders. Sequence transformations [21] may be used to improve the performance of asymptotic series.

An error bound of the remainder in this expansion follows from [13]. We have for $n=0,1,2, \ldots$

$$
K_{i a}(x)=\left(\frac{\pi}{2 x}\right)^{1 / 2} e^{-x}\left[\sum_{k=0}^{n-1} \frac{(i a, m)}{(2 x)^{m}}+R_{n}(a, x)\right],
$$

where the remainder satisfies the simple bound

$$
\left|R_{n}(a, x)\right| \leq 2 e^{\left(a^{2}+\frac{1}{4}\right) / x} \frac{|(i a, n)|}{(2 x)^{n}} .
$$

For a given pair $\{a, x\}$ and precision $\varepsilon$, it is easy to check if a number $n$ can be found such that $\left|R_{n}(a, x)\right|<\varepsilon$.

\section{Continued Fraction}

The Bessel function $K_{v}(x)$ can be expressed in terms of confluent hypergeometric functions. If $z_{n}(x)=U\left(v+\frac{1}{2}+n, 2 v+1,2 x\right)$, then $K_{v}(x)$ can be written as

$$
K_{v}(x)=\pi^{1 / 2}(2 x)^{\prime \prime} e^{-x} z_{0}(x)
$$

where we consider purely imaginary orders $\nu=i a$

The functions $z_{n}$ satisfy the three-term recurrence relation

$$
z_{n-1}(x)=b_{n} z_{n}(x)+a_{n+1} z_{n+1}(x),
$$

with

$$
a_{n+1}=-\left[(n+1 / 2)^{2}-v^{2}\right] \quad b_{n}=2(n+x)
$$

from which a continued fraction representation for the ratio $z_{1} / z_{0}$ can be given:

$$
\frac{z_{1}}{z_{0}}=\frac{1}{b_{1}+} \frac{a_{2}}{b_{2}+} \ldots
$$


The evaluation of $z_{0}(x)$ can be made using the normalization condition ([19])

$$
\sum_{n=0}^{\infty} C_{n=n}=\left(\frac{1}{2 x}\right)^{1+1 / 2}
$$

with

$$
C_{n}=\frac{(-1)^{n}}{n !} \frac{\Gamma(v+1 / 2+n)}{\Gamma(v+1 / 2-n)}
$$

Defining

$$
S=\sum_{n=1}^{\infty} C_{n} \frac{z_{n}}{z_{0}}
$$

we can evaluate $z_{0}$ by means of

$$
z_{0}=\left(\frac{1}{2 x}\right)^{v+1 / 2} \frac{1}{1+S}
$$

The successive terms in the sum (23) can be obtained from the successive approximants to the continued fraction (20). This scheme follows closely the one described in [15] and [20].

The derivative can be obtained from the relation (3) and a relation between contiguous confluent hypergeometric functions. We get

$$
K_{v}^{\prime}(x)=-\frac{K_{v}(x)}{x}\left[1 / 2+x+\left(v^{2}-1 / 4\right) \frac{z_{1}}{z_{0}}\right] \text {, }
$$

where $z_{1} / z_{0}$ is evaluated through the CF (20).

\section{Integral Representations}

In [18] paths of steepest descent for the following integral representations of $K_{i a}(x)$ and $K_{i a}^{\prime}(x)$ were given,

$$
\begin{aligned}
& K_{i a}(x)=\int_{0}^{\infty} e^{-x \cosh t} \cos a t d t \\
& K_{i a}^{\prime}(x)=-\frac{1}{2} \int_{-\infty}^{\infty} e^{-x \cosh t+i a t} \cosh t d t .
\end{aligned}
$$

Two cases have to be considered:

(a) $0 \leq a \leq x, x>0$ (the monotonic case). In this case the appropriate steepest descent path yields the integral representations ([18], Eqs. (2.7) and (4.2)),

$$
\begin{aligned}
& K_{i u}(x)=\int_{0}^{\infty} e^{-x \cosh \tau \cos \sigma-a \sigma} d \tau \\
& K_{i u}^{\prime}(x)=\frac{1}{2} \int_{-\infty}^{\infty} \frac{(\sin \theta \sin \sigma-\cosh \tau)}{\cos \sigma} e^{-x \cosh \tau \cos \sigma-u \sigma} d \tau,
\end{aligned}
$$

where $a=x \sin \theta$ and $\sin \sigma=\left(\sin \theta \frac{\tau}{\sinh \tau}\right)$. 
In applications, and also for numerical calculations (in particular when the parameters $a$ or $x$ are large), it may be important to normalize the integrals by splitting off the dominant exponential term at $\tau=0$. We have

$$
\begin{gathered}
K_{i u}(x)=e^{-\xi} \int_{0}^{\infty} e^{-. x \Phi(\tau)} d \tau, \\
K_{i a}^{\prime}(x)=-e^{-\xi} \int_{0}^{\infty}\left[\cos \theta+\frac{\cosh \tau-1+2 \sin ^{2} \frac{1}{2}(\theta-\sigma)}{\cos \sigma}\right] e^{-.1 \Phi(\tau)} d \tau,
\end{gathered}
$$

where, because $\sigma=\theta$ if $\tau=0$,

$$
\xi=x \cos \theta+a \theta
$$

and

$$
\Phi(\tau)=x[(\cosh \tau-1) \cos \sigma+(\cos \sigma-\cos \theta)+\sin \theta(\sigma-\theta)]
$$

To achieve stable computations for small values of $\tau$ we need to take care of the term $\cosh t-1$, which is quite simple, and of $\theta-\sigma$. The latter follows from

$$
\sin (\theta-\sigma)=\frac{\sin \theta}{\cos \theta \frac{\tau}{\sinh \tau}+\cos \sigma}\left[1-\frac{\tau^{2}}{\sinh ^{2} \tau}\right] .
$$

This requires, for example, a stable algorithm for computing $\sinh \tau-\tau$ for small values of $\tau$, which is also easy to develop.

(b) $0<x<a$ (the oscillatory case). The nonoscillating integral representations are given by Eqs. (3.3), (4.4), and the equation before (3.5)) in [18],

$$
\begin{aligned}
K_{i u}(x)= & e^{-u \pi / 2 M i}\left\{e ^ { - i x } \left[\int_{\tau_{0}}^{x} e^{-\Psi(\tau)}\left(1+i \frac{d \sigma}{d \tau}\right) d \tau\right.\right. \\
& \left.\left.-\frac{1}{1-e^{-2 \pi u}} \int_{\frac{T}{2}}^{\frac{5 \pi}{2}} e^{-\Psi(\tau)}\left(\frac{d \tau}{d \sigma}+i\right) d \sigma\right]\right\} \\
K_{i u}^{\prime}(x)= & e^{-u \pi / 2 \cdot) i}\left\{e ^ { - i x } \left[\int_{\tau_{1}}^{\infty} e^{-\Psi(\tau)}(A(\tau)+i C(\tau)) d \tau\right.\right. \\
& \left.\left.-\frac{1}{1-e^{-2 \pi u}} \int_{\frac{\frac{\pi}{2}}{2}}^{\frac{5 \pi}{2}} e^{-\Psi(\tau)}(B(\tau)+i D(\tau)) d \sigma\right]\right\},
\end{aligned}
$$

where $\chi=x \sinh \mu-a \mu, \tau_{0}=\mu, \cosh \mu=\frac{a}{x}, \mu>0$,

$$
\Psi(\tau)=x \cosh \tau \cos \sigma+a\left(\sigma-\frac{1}{2} \pi\right) .
$$

which differs from Eq. (3.4) in [18] by the last term, given that the factor $e^{-u \pi / 2}$ appears in front of Eqs. (35), (36), and

$$
\sin \sigma=\frac{(\tau-\mu) \cosh \mu+\sinh \mu}{\sinh \tau}
$$


The functions $A(\tau), B(\tau), C(\tau)$, and $D(\tau)$ are given by

$$
\begin{aligned}
& A(\tau)=-\cosh \tau \cos \sigma+\sinh \tau \sin \sigma \frac{d \sigma}{d \tau}, \quad B(\tau)=A(\tau) \frac{d \tau}{d \sigma}, \\
& C(\tau)=-\sinh \tau \sin \sigma-\cosh \tau \cos \sigma \frac{d \sigma}{d \tau}, \quad D(\tau)=C(\tau) \frac{d \tau}{d \sigma}
\end{aligned}
$$

The improper integrals can be straightforwardly computed in terms of the variable $\tau$ by using a standard quadrature rule for improper integrals [14]. The only point to take into account is the accurate computation of the derivative $d \sigma / d \tau$, as we will discuss later. The integrals over a finite interval present a series of numerical features which deserve a careful analysis. Let us consider in detail the evaluation of the corresponding integrals for the function $K_{i u}(x)$. For $K_{i a}^{\prime}(x)$ the same arguments apply.

Let us then consider the evaluation of

$$
\begin{aligned}
K_{i a}^{f}(x) & =-\Re\left[e^{-i \chi} \frac{1}{1-e^{-2 \pi a}} I(x)\right] \\
I(x) & =e^{-\pi a / 2} \int_{\pi / 2}^{5 \pi / 2} e^{-\Psi(\tau)}\left(\frac{d \tau}{d \sigma}+i\right) d \sigma,
\end{aligned}
$$

with $\tau$ related to $\sigma$ through Eq. (38). In principle one can as well evaluate the integral $I(x)$ with respect to $\tau$ which seems simpler than the integration with respect to $\sigma$ given that $\sigma$ can be directly obtained from $\tau$ by applying (38); see Fig. 1 . However, the derivative $d \sigma / d \tau$ would appear in the integral, which goes to infinity as $\tau \rightarrow \tau_{1}, \tau_{1}$ being the $\tau$-value corresponding to $\sigma=3 \pi / 2$. Then it seems safer to integrate with respect to $\sigma$ even if this requires numerical inversion of (38). We will see how a combination of $\tau$ and $\sigma$ integration will lead to a satisfactory implementation of the integral $I(x)$. Before this, let us again rewrite the integral in a more suitable way for computation. By noticing that the functional relation $\tau(\sigma)$ defined by (38) is symmetric around $3 \pi / 2$,

$$
\tau(3 \pi / 2-\theta)=\tau(3 \pi / 2+\theta),
$$

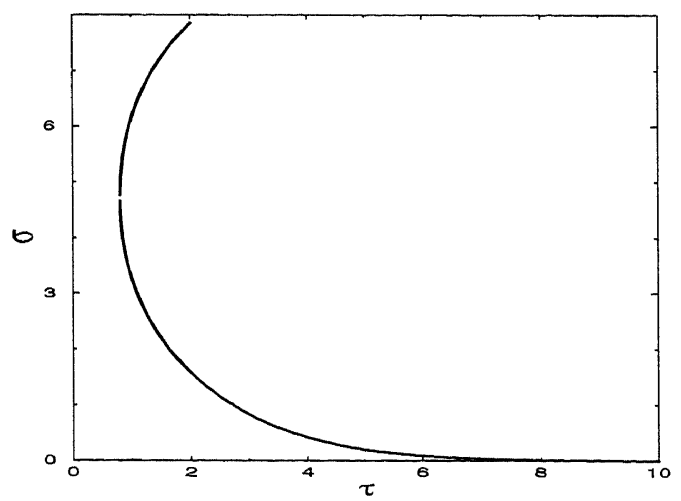

FIG. 1. Representation of $\sigma$ as a function of $\tau$ for $\mu=2$. 
we can write

$$
I(x)=2 e^{-\frac{3 \pi}{2} a} \int_{\pi / 2}^{3 \pi / 2}\left[\sinh \rho \frac{d \tau}{d \sigma}+i \cosh \rho\right] d \sigma
$$

with $\rho=-x \cosh \tau \cos \sigma+a\left(\frac{3 \pi}{2}-\sigma\right)$.

As a first attempt, we can consider the evaluation of the integral with respect to $\sigma$ as it stands. Both the $\rho$ function and the derivative

$$
\frac{d \tau}{d \sigma}=\frac{\sinh ^{2} \tau \cos \sigma}{(\mu-\tau) \cosh \tau \cosh \mu-\sinh (\mu-\tau)}
$$

are continuous and bounded in $[\pi / 2,3 \pi / 2]$. However, the derivative as expressed in (42) is not suitable for numerical computation, given the vanishing of both the numerator and the denominator when $\sigma \rightarrow \pi / 2$ (and subsequently $\tau \rightarrow \mu$ ). A numerically sounder expression for the derivative in the interval $[\pi / 2,3 \pi / 2]$ is

$$
\frac{d \tau}{d \sigma}=\frac{\sinh \tau}{F(\tau-\mu)-\cosh \mu \cosh \tau} \sqrt{E(\tau, \mu)}
$$

with

$$
E(\tau, \mu)=F(\tau-\mu)^{2}-1+\sinh ^{2} \mu\left(2 F(\tau-\mu)^{2}-1\right)+2 \sinh (2 \mu) G(2(\tau-\mu))
$$

and

$$
F(x)=\frac{\sinh x}{x}, \quad G(x)=\frac{F(x)-1}{x}
$$

In this way, by considering an algorithm to evaluate $F(x)-1$ and $G(x)$, the derivative can be accurately computed when $\tau \rightarrow \mu(\sigma \rightarrow \pi / 2)$. In fact, we see that $d \tau /\left.d \sigma\right|_{\sigma=\pi / 2}=-1$.

For integrating with respect to $\sigma$, we need to perform an accurate numerical inversion of (38) for obtaining $\tau(\sigma)$. Hence, we should find the roots (given $\sigma$ ) of $f(\tau)=\sin \sigma \sinh \tau-$ $\tau \cosh \mu-\sinh \mu+\mu \cosh \mu$.

A simple analysis shows that given a positive value for $\sin \sigma, f(\tau)$ has two roots, the smallest one corresponding to values of $\sigma$ in $[\pi / 2, \pi]$ and the largest to $[0, \pi / 2]$ (see Fig. 1 ). As $\sin \sigma \rightarrow 0^{+}$the largest root tends to $+\infty$ and the smallest to $\mu-\tanh \mu$; on the contrary, as $\sin \sigma \rightarrow 1$ the two roots approach each other, being the root for $\sigma=\pi / 2$, a double root. For the numerical inversion of (38), solving $f(\tau)=0$ near degenerate roots (as $\sigma \rightarrow \pi / 2$ ) is bad news, and one can expect difficulties in evaluating with high accuracy the roots corresponding to $\sigma \in[\pi / 2, \pi]$.

On the other hand, for any fixed $\sin \sigma<0$, there is a single root of $f(\tau)$; this is as expected given that $\tau(3 \pi / 2-\theta)=\tau(3 \pi / 2+\theta)$. Numerical inversion for $\sigma \in[\pi, 3 \pi / 2]$ does not present any difficulty and one can, for instance, evaluate the roots for the different $\sigma$ s by using a Newton method with starting value $\tau(\pi)=\mu-\tanh \mu$ (later we will consider a refinement of the method).

For the reasons explained above, we consider

$$
I(x)=\int_{\tau(\pi / 2)}^{\tau(\pi)}\left(\sinh \rho+i \cosh \rho \frac{d \sigma}{d \tau}\right) d \tau+\int_{\pi}^{3 \pi / 2}\left(\sinh \rho \frac{d \sigma}{d \tau}+i \cosh \rho\right) d \sigma,
$$


which allows us to write

$$
\begin{aligned}
K_{i u}^{f}(x)= & \frac{e^{-\pi \omega / 2}}{\sinh \pi a}\left[\int_{\mu-\tanh \mu}^{\mu}\left(\cos \chi \sinh \rho+\sin \chi \cosh \rho \frac{d \sigma}{d \tau}\right) d \tau\right. \\
& \left.-\int_{\pi}^{.3 \pi / 2}\left(\cos \chi \sinh \rho \frac{d \tau}{d \sigma}+\sin \chi \cosh \rho\right) d \sigma\right]
\end{aligned}
$$

where, given a value of $\sigma$ in the second integral, we obtain $\tau$ by using a Newton method for the function $f(\tau)$. This numerical inversion can be done parallel to the integration, using as first starting value $\mu-\tanh \mu$ and subsequently using as starting values the previously evaluated root. The sequences of roots obtained in this way form a decreasing sequence of values of $\tau$ for increasing values of $\sigma$ in $[\pi, 3 \pi / 2]$. Applying the successive Newton steps in this way guarantees a fast convergence.

The evaluation of $K_{i a}^{\prime}(x)$ for the oscillatory case is very similar to the evaluation of $K_{i a}(x)$. We start from the integral representation (36). Again, the improper integral can be evaluated as it stands, while the rest of integrals can be put into the form

$$
K_{i a}^{\prime f}(x)=-m\left[e^{-i x} \frac{e^{-\pi u / 2}}{\sinh \pi a} \int_{\pi / 2}^{3 \pi / 2}(B(\tau) \cosh \rho+i D(\tau) \sinh \rho) d \sigma\right]
$$

which gives

$$
\begin{aligned}
K_{i a}^{\prime f}(x)= & \frac{e^{-\pi u / 2}}{\sinh \pi a}\left[\int_{\mu-\tanh \mu}^{\mu}(\cos \chi \cosh \rho A(\tau)+\sin \chi \sinh \rho C(\tau)) d \tau\right. \\
& \left.-\int_{\pi}^{3 \pi / 2}(\cos \chi \cosh \rho B(\tau)+\sin \chi \sinh \rho D(\tau)) d \sigma\right] .
\end{aligned}
$$

\section{NUMERICAL DISCUSSION}

We compare the different methods we have discussed for the evaluation of $K_{i a}(x)$ and $K_{i l}^{\prime}(x)$ (series expansion, continued fraction, asymptotic expansion, and nonoscillating integral representation) for a precision of $10^{-9}$. For this purpose, we compare the nonoscillating integrals with the other approaches and the continued fraction with the series and the asymptotic expansion. We will explicitly show the performance of the different approaches for $K_{i a}(x)$; for its derivative the same comparative results are found.

Figure 2 compares the results obtained via CF method and via series expansions. The region of coincidence is rather restricted, showing that the regions of validity are in some sense reciprocal. Series expansions are expected to work better as $x$ becomes smaller and as $a$ becomes larger; this fact can be understood from Eq. (9).

The range of validity of the continued fraction method is extended compared with the case of real orders. The reason for this can be found in the expression for $a_{n+1}$ when compared to the real case (Eq. (19)). The region of validity for the CF method is large enough to completely cover the region of validity of the asymptotic expansion for large $x$ (compare Figs. 3 and 4). In fact, the asymptotic expansion is of more restricted applicability than for the case of real order, given that the coefficients in the expansion become greater, for a same 


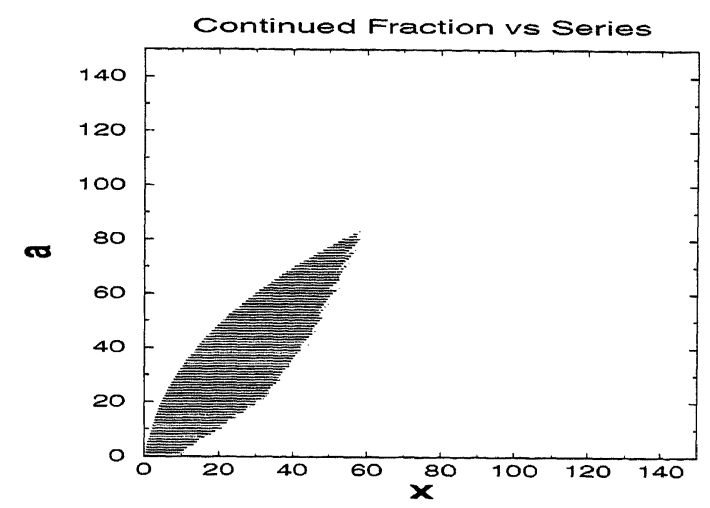

FIG. 2. Comparison between the evaluation of $K_{i d}(x)$ via continued fraction and via series expansion for a precision better than $10^{-4}$. The $x-a$ plane is scanned with point spacing $\Delta a=1, \Delta x=1$. The points where the coincidence between both approaches is at least of nine significant digits are shown.

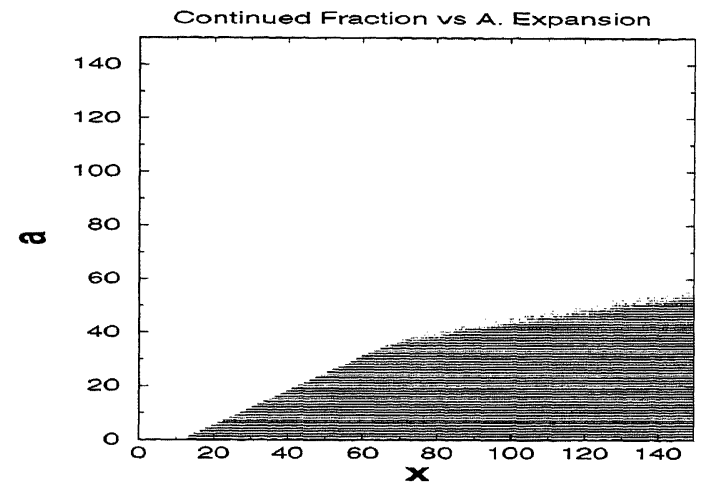

FIG. 3. Same as Fig. 2, but for the comparison between the evaluations via continued fraction and via asymptotic expansion. The points of coincidence within a precision better than $10^{-4}$ are plotted.

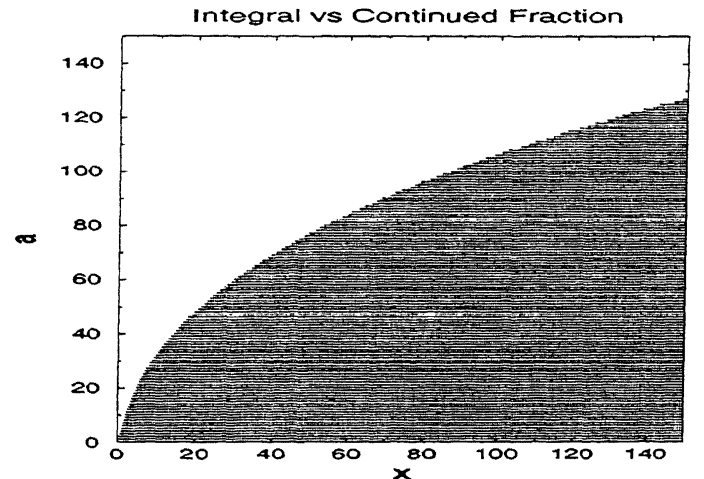

FIG. 4. Comparison between the evaluations via continued fraction and via nonoscillating integral representation. The points of coincidence for a precision better than $10^{-4}$ are plotted. 


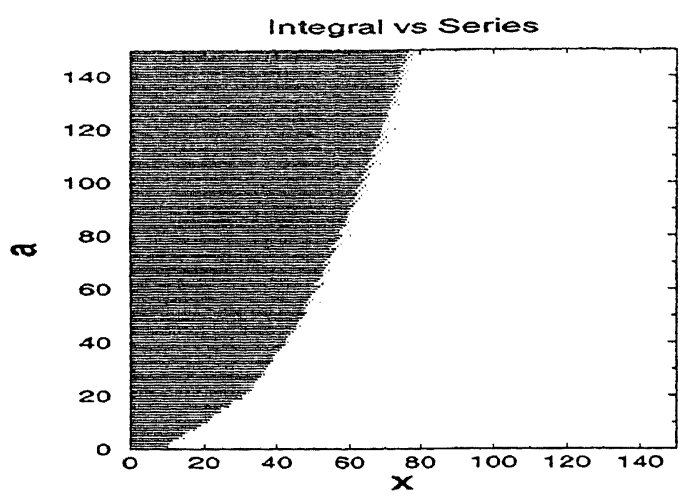

FIG. 5. Comparison between the evaluations via series expansion and via nonoscillating integral representation. The points of coincidence within a precision better than $10^{-9}$ are plotted.

value of $|v|$ when $v$ is purely imaginary (Eq. (9)). The use of the asymptotic expansion may be of interest only for reasons of speed, when the expansion succeeds in giving enough accuracy.

In Figs. 4 and 5 we compare the nonoscillating integrals with the $\mathrm{CF}$ method and series expansions. The regions plotted are the regions of validity of series and CF method which are enough for an evaluation for $10^{-9}$ precision for $x<60$ (Fig. 8) and orders smaller than 200. For larger orders, underflow problems in the evaluation of the function appear when a minimal number of $10^{-300}$ is permitted by the computer (usual restriction).

Numerical experiments show that the computation of all the integrals, except the integral over $\tau$, is well behaved and that Piessens' algorithm for the improper integral together with a 20-point Gauss-Legendre quadrature for the $\sigma$ integral over finite intervals gives an accuracy better than $10^{-9}$. However, the $\tau$-integral keeps the information regarding the "singularity" of the integration path as $\mu \rightarrow 0$ and as $\mu$ becomes large, and we should consider a higher order quadrature of this integral, in particular, near the line $a=x$ (with $a>x)$. In the figures shown, we have scanned the $(a, x)$ plane with regularly spaced points, being the closest points to the line $a=x$ those for which $a=x+0.1$. In this case, a 60-point Gauss-Legendre for the $\tau$ integration does the job for such points.

The problem with the $\tau$ integral as $a \rightarrow x^{+}$comes from a very fast increase of the integrand near the lower integration limit, and it is related to the peculiar limiting form of the integration path (see Figs. 6 and 7). Properly dividing the integration interval helps in improving the precision with less Gauss points. Integrating in terms of $\sigma$ does not particularly help, given the problems that the numerical inversion in order to evaluate $\tau(\sigma)$ would encounter. The integration path approaches the positive $\sigma$ semi-axis, and it is tempting to believe that the problem is solved by first integrating over $\tau$ in the interval $[\mu,+\infty)$, and doing the rest of integration over $\sigma$; however, we need accurate numerical inversion in this last case. Given that the path for this last integration is almost a straight vertical line (with $\tau$ as the y axis) for any given $\sigma$ we have nearly the same value of $\tau$. This is a delicate situation for accurately computing such values of $\tau$, particularly for $\sin \sigma \simeq 1$, for which there is nearly a double root.

In spite of the accuracy problems for the integrals in the oscillatory case when $a \simeq x$, we observe from Fig. 8 that the CF, together with the series expansion and the integral 


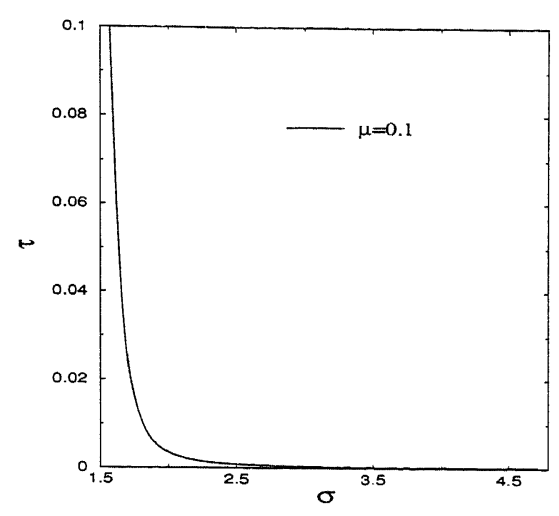

FIG. 6. Representation of $\tau$ as a function of $\sigma$ for $\mu=0.1$.

representation for the oscillatory case, are enough to build an algorithm which gives a precision of $10^{-9}$ for $x<100$ and any real order, limited by an underflow number of $10^{-300}$ $(\nu \sim 200)$. This underflow takes place, for instance, when evaluating $f_{0}(12)$ for the series. Similar problems happen for the integrals. With this restriction $(x<100)$ one can use the CF and McLaurin series for the case $a>x$ with $a$ close to $x$ (Fig. 8), therefore avoiding the use of integrals in the conflictive region. A 20-point Gauss-Legendre is then sufficient to evaluate all integrals over finite intervals with an accuracy of $10^{-9}$.

Uniform asymptotic expansions could be considered for the computation of $K_{i a}(x)$ when the computations via CF, series, and integrals fail (large parameters, see Fig. 8). This will be discussed in a later paper.

Of course, in order to build an efficient algorithm, the speed of the different methods has to be taken into account: In the region of coincidence of series and continued fraction, series are faster (about a factor 2) than the continued fraction method. On the other hand, the speed of continued fraction and asymptotic expansion are similar in the region of coincidence of both approaches shown in Fig. 3. Finally, the speed of the calculation of the nonoscillatory integrals is slower than any of the other approaches; so, they have to be used only in case the other approaches fail to converge, as indicated in Fig. 8.

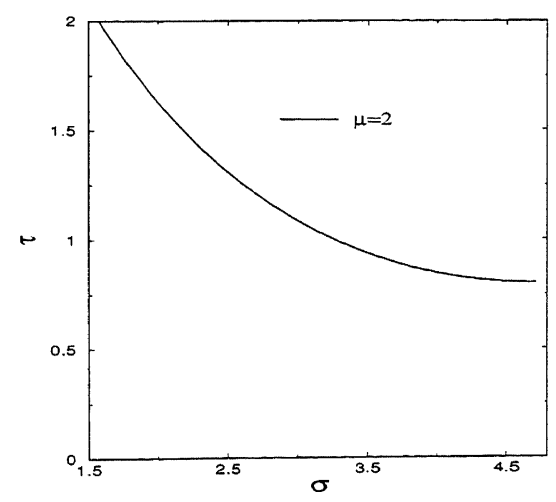

FIG. 7. Representation of $\tau$ as a function of $\sigma$ for $\mu=2$. 


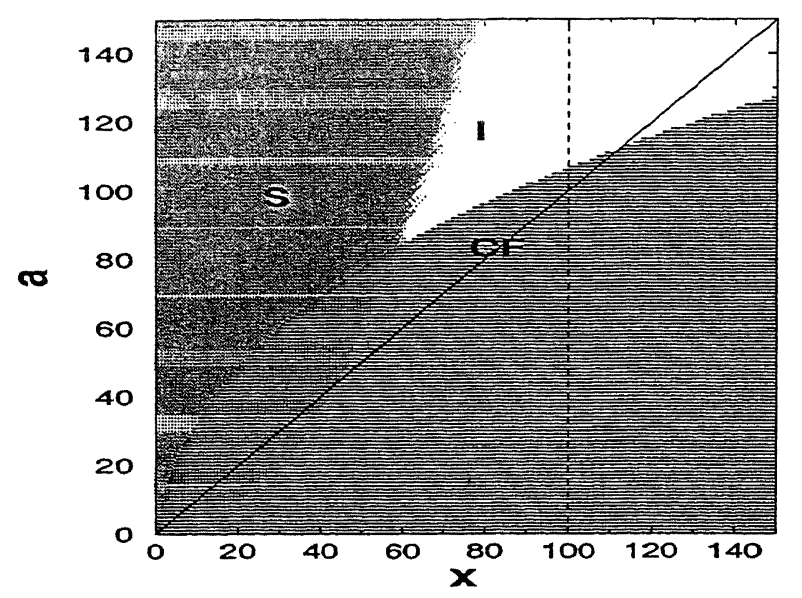

FIG. 8. Regions where the different approaches are applied for an accuracy of $10^{-9}$ : series expansion ( $\mathrm{s}$ ), continued fraction method (CF), and integrals (I). The lines $a=x$ and $x=100$ are also shown. The unshaded region is covered by integrals, except for a tiny region above the line $a=x$. In the region of coincidence of series and $\mathrm{CF}$, series converge faster. Series, the integral representation for the oscillatory case and the CF method, are enough to compute $K_{i u}(x)$ with a precision of $10^{-9}$ when $x<100$.

In summary, the different methods of computation for a precision better than $10^{-9}$ in the region of parameters $0<a<200,0<x<100$, are the following:

1. Series for moderate $x$. A fit to Fig. 8 shows that series can be used when $a>x^{2} / 40$.

2. Continued fraction method for moderate $a$. When $x>a^{2} / 110$, we use the CF method if series cannot be applied.

3. Integral representations are used in the rest of the parameter space, in particular the integral for the oscillatory case.

\section{ACKNOWLEDGMENTS}

A. Gil and J. Segura acknowledge financial support from Generalitat Valenciana (GV99-146-1-04). The authors thank the referees for valuable suggestions.

\section{REFERENCES}

1. M. Abramowitz and I. A. Stegun, Eds., Handhook of Mathematical Functions (National Bureau of Standards; reprinted by Dover, New York, 1972).

2. J. P. Boris and E. S. Oran, Numerical evaluation of oscillatory integrals such as the modified Bessel function $K_{i \zeta}(x)$.J. Comput. Phys. 17, 425 (1975).

3. LI. Closas and J. Fernández Rubio, Calculo rapido de las funciones de Bessel modificadas $I_{i,}(X)$ y sus derivadas. Stochastica 11, 53 (1987).

4. W. J. Cody and K. E. Hillstrom, Chebyshev approximations for the Coulomb phase shift. Math. Comput. 24, 671 (1970).

5. T. M. Dunster, Uniform asymptotic solutions of second-order linear differential equations having a double pole with complex exponent and a coalescing turning point. SIAM J. Math. Anal. 21, 1594 (1990).

6. T. M. Dunster, Conical functions with one or both parameters large. Proc. Rol. Soc. Edinburgh 119A, 311 (1991). 
7. U. T. Ehrenmark, The numerical inversion of two classes of Kontorovich-Lebedev transform by direct quadrature. J. Comput. Appl. Math. 61, 43 (1995).

8. W. Gautschi, Computational aspects of three-term recurrence relations. SIAM Rev. 9, 24 (1967).

9. M. K. Kerimov and S. L. Skorokhodov, Calculation of modified Bessel functions in the complex domain. Comput. Math. Math. Phys. 25, 15 (1984). (Translation from Zh. Vychisl. Mat. Mat. Fiz. 24, 650 (1984).)

10. T. Kiyono and S. Murashima, A method of evaluation of the function $K_{i,}(x)$. Mem. Fac. Eng. Kyoto Univ. 35 , 102 (1973).

11. J. D. Lear and J. E. Sturm, An integral representation for the modified Bessel function of the third kind, computable for large, imaginary order. Math. Comput. 21. 496 (1967).

12. N. N. Lebedev, Special Functions and Their Applications (Dover, New York, 1972).

13. F. W. J. Olver, Asymptotics and Special Functions (Academic Press, New York, 1974; reprinted by A. K. Peters), 1997.

14. R. Piessens and E. De Doncker, subroutine DQAGIE (obtained from SLATEC Public Domain Library).

15. W. H. Press, S. A. Teukolsky, W. T. Vetterling, and B. P. Flannery, Numerical Recipes in Fortran 77 (Cambridge Univ. Press, Cambridge, UK, 1992), pp. 239-240.

16. J. M. Rappoport, Canonical vector polynomials for the computation of the complex order Bessel functions with the tau method. Comput. Math. Appl. 41, 399 (2001).

17. N. M. Temme, Special functions: An Introduction to the Classical Functions of Mathematical Physics (Wiley, New York, 1996).

18. N. M. Temme, Steepest descent paths for integrals defining the modified Bessel functions of imaginary order. Meth. Appl. Anal. 1, 14 (1994)

19. N. M. Temme, On the numerical evaluation of the modified Bessel function of the third kind. J. Comput. Phys. 19, 324 (1975).

20. I. J. Thompson and A. R. Barnett, Modified Bessel functions $I_{v}(z)$ and $K_{v}(z)$ of real order and complex argument, to selected accuracy. Comput. Phys. Commun. 47, 245 (1987).

21. E. J. Weniger and Čížek, J., Rational approximations for the modified Bessel functions of the second kind. Comput. Phys. Commun. 59, 471 (1990). 